Gynäk. Rdsch. 1984;24(suppl. 2):I-VI

\title{
Contents, Vol. 24, Supplement 2, 1984
}

Inhalt

Editorial $\quad 1$

Vorwort 3

1. Hauptthema: Amenorrhoe

Hauptreferat

Tscherne, G.: Amenorrhoe 5

Vorträge zum 1. Hauptthema

Fischl, F.; Deutinger, J.: Zuweisungsdiagnose: Amenorrhoe 10

Huber, J, C.; Schurz, Brigitte; Stempel-Smekal, Gabriela; Metka, M.; Knogler, W.;

Spona, J.: Erweiterte endokrine Abklärung bei primärer und sekundärer Ame

norrhoe

13

Urdl, W.; Kowatsch, A.; Schweditsch, M. O.; Tscherne, G.: Die Behandlung der

hypothalamischen Amenorrhoe mit pulsatiler (GNRH)-Gabe 14

Kowatsch, A. W.; Tscherne, G.; Urdl, W.: Die sekundäre hypergonadotrope Ame

norrhoe - Klimakterium präcox 18

Urdl, W.; Schweditsch, M. O.; Kowatsch, A.: Androgenisierung - androgenetische

Amenorrhoe und sexualhormonbindendes Globulin (SHBG)

Stempel-Smekal, G.; Knogler, W.; Schurz, B.; Huber, H.; Spona, J.: Die Amenor

rhoe post Pille

23

2. Hauptthema: Forzeps versus Vakuum

Hauptreferat

Reinold, E.: Forzeps versus Vakuum 25

Inhalt

IV

Koreferat Endler,M.; Bartl, W.; Gruber, W.: Vakuumextraktion versus Forzepsentbindung ... 32

3. Hauptthema: Sectio caesarea

Hauptreferat

Bichler, A.: Sectio caesarea 36

Koreferat

Gruber, W.: Neue Aspekte der Sektioindikation

43

Vorträge zum 3. Hauptthema

Beck, A.; Vutuc, Ch.: Die Mortalität und Letalität der Schnittentbindung in Österreich 47

Pateisky, N.; Stempel-Smekal, G.; Reinold, E.: Indikationswandel zur Sectio caesarea 50

Moser, W.; Schausberger, L.; Golob, E.: Zur Problematik der Sectiofrequenz 52

Nagl, F.; Stummvoll, W.; Wiebogen, L.: Kann durch eine Erhöhung der Sectiofre quenz die Azidose-Morbidität gesenkt werden? 56

Mayer, H. O.; Holzer, E.; Lichtenegger, W.; Weiß, P. A. M.: Sectio caesarea bei

Kindern unter 2000 Gramm und EPH-Gestose 
Dadak, Ch.; Lasnik, E.: Schwangerschaft und Geburt nach vorangegangener Sectio caesarea 61

Lahousen, M.;Burmucic, R.: Geburtsverlauf nach Kaiserschnitt 63

Stummvoll, W.; Nagl, F.; Wiebogen, L.: Zur Geburtsleitung nach vorausgegangener

Sectio caesarea unter besonderer Berücksichtigung der mütterlichen und kindli-

chen Morbidität 66

Schmid, R.; Dalbauer, Ch.; Floder, F.: Geburtsleitung nach Section caesarea an einem geburtshilflichenSchwerpunktkrankenhaus 68

Hofmann, H.; Weiß, P. A. M.; Hirschmann, H.; Kainer, F.: Sectio bei Diabetes mellitus $\quad 71$

Klug, P. W.; Mayer, H. G. K.: Zur Uterusnaht bei Schnittentbindung. Ein Bericht über427Fälle 74

Müller-Hartburg, W.; Sedlak, O.: Mütterliche und kindliche Ergebnisse von 20.000

fortlaufenden Entbindungen bei strenger Indikation zur Schnittentbindung .... 76

Endler,M.; Spernol,R.; Gruber, W.: Sterilität nach Sectio 80

Freie Vorträge

Rudelstorfer, R.; Simbruner, G.; Tabsh, K.; Khoury, A.; Wasserstrum, N.;

Janisch, H.; Assali, N. S.: Direkte und indirekte Kalorimetrie der uterofetopla-

zentaren Einheit im Schafmodell 82

Skodler, W. D.; Philipp, K.; Pateisky, N.: Uteroplazentare Durchblutungsmessung bei

kleinen Plazenten 84

Inhalt $\mathrm{V}$

Baumgarten, K.; Neumann, M.: Verschiedene Parameter für die Diagnostik der Plazentainsuffizienz 87

Fuchs, G.; Bali, Ch.; Kritz, H.; Rost, I. M.; Irsigler, K.; Leodolter, S.: Erfahrungen

mit der Insulinpumpe bei schwangeren Diabetikerinnen 91

Rost, I. M.; Bali, Ch.; Fuchs, G.; Irsigler, K.; Leodolter, S,: Fetal Outcome bei 106 insulinpflichtigen Schwangeren 93

Karpellus, E.; Bichler, A.: Diabetes-Screening im Wochenbett: oraler Glukosetoleranztest oder glykosiliertes Hämoglobin A 1? 94

Kowatsch, A.; Rosanelli, K.; Zeichen, H. L.: Schwangerenvorsorge bei sehr kleinen Frühgeborenen. Ergebnisse einer multizentrischen Studie 97

Langer, M.; Salzer, FL: Lungenreifebestimmung aus dem Fruchtwasser: eine neue Methode zur Messung der statischen Oberflächenspannung im Vergleich zur dynamischen Oberflächenspannungsmessung in der Wilhelmy-Waage 98 Bartosch, B.;Fodor, G.: Rheobasemessung und Schwangerschaftsverlauf 101 Lechner, W.;Daxenbichler, G.: Tokolyse mit oder ohne Beta-Blocker? 103 Hellmich, C; Bartosch, B.: Veränderungen im Geburts-CTG beim Liegen, Sitzen, Stehen bzw. Gehen. Ein Beitrag zur telemetrischen Geburtsüberwachung 105 Wiebogen, L.; Nagl, F.; Stummvoll, W.: Zur Qualität des subpartalen Kardiotokogramms: Externe Ableitung mit Ultraschall und Autokorrelation im Vergleich mit direktem fetalen Elektrokardiogramm 107

Sevelda, P.; Reinold, E.: Entbindungen bei Patientinnen mit Hypertonie 109 Dadak, Ch.; Lasnik, E.: Betreuung der Frau in der Gravidität in der Altersgruppe über35Jahre 111

Vutuc, Ch.; Beck, A.: Zum Problem der Transferierung in der Geburtshilfe 113 
Genger, FL; Sevelda, P.; Salzer, FL: Gehen in der Eröffnungsperiode. Erste Ergebnis se einer randomisierten Vergleichsstudie 116

Stummvoll, W.; Nesser, H. L: Hypoplastisches Linksherzsyndrom: Pränatale Diagno stik mittels zweidimensionaler Echokardiographie 118

Anderl, P.; Hetzenauer A.: Choledochuszyste als Komplikation in der Schwanger-

schaft 120

Hamann, B.; Büchner, Ch.; Löffler, Ch.: Erfahrungsbericht über das Medusa-Pessar

MPL-Cu-240-Ag 122

Hamann, B.; Jorde A.; Löffler Ch.: Erfahrungsbericht über die Anwendung von

Stützpessaren als ,unblutige Cerclage-Operationen” bei Zervixinsuffizienz .... 124

Moser, W.; Golob, E.: Klinische Aspekte des Intrauterinpessars 125

Bartl,W.; Bernaschek, G.: Zur Diagnose von Mammakarzinomenim Ultraschall . . 129

Arns, H.-H.: Kontaktthermographie und Frühdiagnostik der Intervall-Karzinome ... 131

Pfersmann, Ch.; Bartl, W.; Wolf, G.: Die Validität der thermographischen, mammographischen und sonographischen Untersuchung bei histologisch verifizierten

Mammakarzinomen im Stadium T 1133

Strigl, R.; Fuchs, H. D.; Kleinhans, M.; Loos, W.; Graeff H.: Zur Frage der präoperativen Markierung von klinisch nicht palpablen Veränderungen der

Brust 135

Hengstberger, M.: Verbesserte Brustkrebsvorsorge - Hydropalpation durch Blinde als Alternative zur Selbstkontrolle $\quad 137$

Inhalt

VI

Bartl, W.; Euller, A.; Pfersmann, Ch.; Janisch, H.: Wandel therapeutischer Wege bei

Mammakarzinomen im Laufe der Zeit (unter besonderer Berücksichtigung der

Therapiekonzepte und Ergebnisse des hauseigenen Kollektives im Stadium T 1) . 140 Fischl, F.;

Deutinger, J.; Janisch, H.: Neue Aspekte der homologen Insemination . . 142 Metka, M. M.;

Huber, J. C: Spermaenhancement und intrauterine Insemination .... 144 Metka, M. M.; Huber, J.

C: Modifizierter Hamsterei-Spermien-Penetrationstest

durch Verwendung der Fluoreszenzmikroskopie 147

Szalay, S.; Spernol, R.; Nachtigall, M.: Die In-Vitro-Fertilisierung in Klagenfurt .... 149 Freude, G.; Zeibekis, N.; Boschitsch, E.; Leodolter, S.: Second Look Laparoskopien

nach Sterilitätsoperationen 150

Boschitsch, E.; Leodolter, S.: Der Kinderwunschpaß - eine neue Form der Dokumen-

tation in der Sterilitätssprechstunde 152

Lichtenegger, W.; Lahousen, M.; Pickel, M.: Die Radikaloperation beim Ovarial-

kärzinom 153

Pateisky, N.; Gitsch, E.; Philipp, K.; Skodler, D.: Erste Ergebnisse der Radioimmun-

szintigraphie im Rahmen der operativen Behandlung des Ovarialkarzinoms .... 155 Szepesi, T.;

Kärcher, H. K.; Stadler, B.; Hawliczek, R.; Janisch, H.; Schieder, Ch.;

Nowotny, Ch.; Dadak, Ch.: Interdisziplinäres Therapiekonzept des Ovarialkarzi

noms aus strahlentherapeutischer Sicht 157

Bieglmayer, Ch.; Schieder, K.; Csaicsich, < P.; Nowotny, Ch.: Tumormarker für Ova-

rialkarzinome: Erste Erfahrungen mit CA $125 \quad 160$

Schieder, K.; Csaicsich, P.; Kölbl, H.; Nowotny, Ch.; Bieglmayer, Ch.; Breitenecker, G.; Szalay, St.; Szepesi, T.; Janisch, H.: Erste Erfahrungen mit dem Tumor- 
stammzellassay als Methode zur prätherapeutischen Zytostatikasensitivitätstestung beim Ovarialkarzinom 163

Fuith, L. C; Dworzak, E.; Zwierzina, D.; Grunicke, H.; Dapunt, O.: Veränderungen der Amylase-Isoenzyme im Serum von Patientinnen mit Ovarialkarzinomen . . . 165 Nowotny, Ch.; Szepesi, T.: Koordination der Betreuung von Ovarialkarzinom-Patientinnen zwischen niedergelassenen Ärzten und Klinik 168 Gitsch, E.; Pateisky, N.; Philipp, K,; Skodler, D.: Die Radioisotopenradikaloperation mit einem tumorassoziierten Antikörper 170

Bernaschek, G.; Tatra, G.; Janisch, H.: Die Vorteile der rektalen Sonographie bei der Rezidivdiagnostik des Zervixkarzinoms 171

Wiborny, R.; Friedrich F.: Auftreten von gynäkologischen Malignomen in einem ländlichen Gebiet 173

Breitenecker, G.; Holzner, J. H.: Zur Entwicklung der gynäkologischen Zytodiagnostik in Österreich seit dem Jahre 1969 174

Lahodny, J.: Korporofundale partielle periphere Blasendenervation - ein Konzept zur chirurgischen Sanierung der Dranginkontinenz 177

Schurz, A. R.; Gschliesser, H.: Die Behandlung des Scheidenblindsackvorfalls durch Vaginaefixatio sacrospinalis nach Richter 179

Hosmann, J.; Stary, A.; Söltz-Szöts, J.; Salzer, H.: Chlamydien- und Mycoplasmeninfektionen bei der chronischen Adnexitis 181

Bernaschek, G.; Lubec, G.; Schaller, A.: Uterus- und Ovargröße im Kindesalter eine echographische Studie 183

Swolin, K.: Elektro-Mikros-Chirurgie - Swolintechnik in der Gynäkologie 185

Autorenregister 188 